\title{
VASCULITIS TRAS LA MONITORIZACION DE PRESIÓN ARTERIAL
}

\section{VASCULITIS AFTER BLOOD PRESSURE MONITORING}

Olga Ventura Ribes ${ }^{\mathrm{a}}$, Francisco Herrero Machancoses ${ }^{\mathrm{b},}$ Jesús Rosel Remírez $^{\mathrm{b}}$.

${ }^{a}$ Hospital de la Plana, Ctra. de Vila-real a Burriana, Km 0.5. 12540 Vila-real, Castellón.

${ }^{\mathrm{b}}$ Universitat Jaume I, Facultat de Ciències de la Salut. Av. de Vicent Sos Baynat, s/n, 12071 Castelló de la Plana.

Número de palabras: 1167

Autor de contacto: Olga Ventura Ribes. C $\backslash$ Karl Raimund popper, 1, $1^{\circ} \mathrm{e} .12003$, Castellón de la Plana (Castellón). olventu@gmail.com

Conflicto de intereses: No existen

Fuentes de Financiación: Ninguna

Éste trabajo no ha sido presentado a ningún congreso o reunión científica, y no ha recibido ningún premio o mención. 


\section{RESUMEN}

\section{Introducción}

Descripción de aparición equimosis en un brazo asociado inicialmente a la toma de presión arterial, por Vasculitis Leucocitoclástica y propuesta de utilidades alternativas de la toma de presión arterial.

\section{Caso Clínico}

Varón de 80 años acude a urgencias con disnea, insuficiencia cardíaca, insuficiencia renal prediálisis con hiperpotasemia e inestabilidad hemodinámica. Durante su estancia desarrolló una lesión cutánea con aspecto de púrpura palpable, marcada desde el límite inferior del manguito de presión arterial hasta la zona distal de la mano y que no desaparecía a la vitropresión, y prurito. Durante su ingreso la lesión del brazo se extendió a todos los miembros, tanto superiores como inferiores

El estudio de la lesión del brazo concluyó con diagnóstico de vasculitis Leucocitoclástica por la presencia de eosinófilos, lo que sugiere probable etiología medicamentosa a un antibiótico que llevaba tomando siete días antes de la entrada a urgencias.

\section{Discusión}

Se plantea la necesidad de monitorización seriada de la presión arterial, y la duración de dicha monitorización en pacientes inestables frente a los efectos secundarios de esta técnica. También el estudio de otras utilidades de la toma de presión arterial.

\section{Palabras Clave}

Vasculitis Leucocitoclástica cutánea, Presión Arterial. 


\section{ABSTRACT \\ Introduction}

Description of ecchymoses appearance in the arm, initially associated with measurement of blood pressure, but caused by leukocytoclastic vasculitis, and proposal for alternative utility of measuring blood pressure.

\section{Clinical Case}

80-year-old male came to ER with dyspnea, heart failure, renal failure predialysis with hyperkalemia and hemodynamic instability. During his stay he developed a skin lesion that looks like palpable purpura, marked from the lower limit of the blood pressure cuff to the distal area of the hand that not disappeared with vitropression, and pruritus. During admission the arm injury was extended to all members, both upper and lower. The study of the arm injury concluded with diagnosis of leukocytoclastic vasculitis given the presence of eosinophils, that which suggested probable drug etiology to an antibiotic that had been taken since seven days prior to admission to ER.

\section{Discusions}

The need for serial monitoring of blood pressure, and the duration of such monitoring in unstable patients considering the side effects of those techniques was questioned. In addition, the study of other utilities of measuring blood pressure.

\section{Keywords}

Cutaneous Leukocytoclastic Vasculitis, Blood Pressure. 


\section{INTRODUCCIÓN}

La toma de presión arterial seriada es una técnica de uso habitual que, sin embargo, puede presentar ciertos riesgos, es por ello que se expone en diversos manuales la necesidad de cambiar el manguito de brazo cada $8 \mathrm{~h}$. En el mismo sentido, la bibliografía describe lesiones traumáticas de la piel por la inadecuada colocación del manguito ${ }^{1,2}$. Por la presión mantenida del mismo se puede presentar magulladuras, escoriaciones, equimosis y ampollas. También quemaduras, si se administran soluciones a través de un catéter situado por debajo del manguito ${ }^{3,4}$. En la aplicación quirúrgica del manguito de isquemia también se recomienda el deshinchado del manguito cada 30 minutos ${ }^{5,6}$. Por todo ello, cuando se presenta una lesión cutánea delimitada por el manguito pueden presentarse dudas acerca de su diagnóstico.

La vasculitis Leucocitosclástica engloba a un grupo heterogéneo de procesos clinicopatológicos que se caracteriza por una inflamación y necrosis de la pared de los vasos. Generalmente se inflama el lumen, por lo que se asocia con isquemia de tejidos. Esta inflamación viene mediada por tres factores inmunológicos: el depósito de inmunocomplejos circulantes, la unión directa del anticuerpo al antígeno presente en la pared de los vasos y la activación leucocitaria a través de anticuerpos dirigidos específicamente contra antígenos leucocitarios (ANCA). El tamaño y la forma de los inmunocomplejos determinan la clase de vaso, según tamaño y órgano afectado, así como la clínica del paciente. Las manifestaciones clínicas son variadas, ya que dependerán del territorio irrigado por el vaso comprometido. Puede tratarse entonces de una enfermedad benigna ${ }^{7} \mathrm{o}$ de otra extraordinariamente grave ${ }^{8}$. 


\section{CASO CLÍNICO}

Ingresa en urgencias hospitalarias, un varón de 80 años remitido a las 00:11h por el médico de atención continuada debido al aumento de su disnea habitual, acompañada de anuria de $24 \mathrm{~h}$. Presenta mal aspecto general, normohidratado, palidez cutánea, consciente y orientado, con respiración superficial y cianosis labial. Sus constantes al ingreso fueron: presión arterial 143/47 mmhg; frecuencia cardiaca 60 por minuto; temperatura $36.2^{\circ} \mathrm{C}$, saturación de oxigeno $52 \%$ y glasgow 15 . Auscultación cardiaca con tonos arrítmicos sin soplos, y la pulmonar con abolición de los murmullos vesiculares y sibilantes. Presenta abdomen globuloso, blando y depresible, sin peritonismos, sin masas ni megalias. Sus extremidades presentan edemas con fóvea hasta la rodilla con pulsos papables. Sin focalidad neurológica central.

Antecedentes personales sin alergias medicamentosas conocidas, totalmente independiente para las actividades de la vida diaria (Barthel 85), ex-fumador de 30 años de 90 paquetes/año, sin hábito enólico. Presenta Diabetes Mellitus tipo 2, diagnosticada en 1975; Hipertensión arterial; no Dislipemias; Hiperuricemia; Insuficiencia cardíaca clase III de la NYHA (ingresado en cardiología previamente en Junio y Agosto de 2013, y Marzo 2014); Estenosis de canal lumbar; Hiperplasia benigna de próstata; Hernia de hiato y Diverticulosis; Insuficiencia renal crónica estadio 3 con cifras de creatinina basales de 1.8-2 mg/dL. Ha sido intervenido de estenosis de canal lumbar y de fístulas perianales.

Como tratamiento habitual está tomando Repaglinida 2mg (1-1-1), Insulina Lantus (230-0), Atorvastatina 10mg (0-0-1), Seguril 40mg (2-1-0), Carduran Neo 8mg (1-0-0), Amlodipino 10mg (1-1-0), Zaldiar (1-1-1), Omeoprazol 20mg (0-0-1), Noctamid 1mg (0-0-1), Adiro 100mg (0-1-0),Alopurinol 100mg (0-1-0), Encorcor 2,5 mg (1-00), Moxon 0.4mg (1-0-0),Ferrogradumet (1-0-0), Losartan 100mg (1-0-0) y Royen $500 \mathrm{mg}(1-0-0)$ 
Al realizar las exploraciones complementarias observamos que, en la radiografía de tórax presenta edema intersticial y edema agudo de pulmón; En el electrocardiograma arritmia cardíaca por fibrilación auricular a $70 \mathrm{mlp}$, QRS 0.12 y bloqueo de rama derecha. Las pruebas de laboratorio muestran un hemograma sin datos relevantes, Creatinina 3.60, Na 132, K 6.6, proBNP 5.308. El resto sin datos relevantes

Se concluye como impresión diagnóstica Edema agudo de pulmón, Insuficiencia renal reagudizada con hiperpotasemia.

Durante su estancia necesitó tratamiento farmacológico con Primperán 10 mgs, Cloruro Mórfico 2mg, Perfusión en bomba de Solinitrina 50mg en 250 a 3 ml/h, Seguril 60mg, Perfusión de Seguril $80 \mathrm{mg}$ en $50 \mathrm{cc}$ de suero fisiológicos a $0.2 \mathrm{ml} / \mathrm{h}$, enema de Resin Calcio 50 gr en 200mL de agua, Novorapid iv 10 UI en 100cc Suero Glucosado al 5\% a 200ml/h y oxígeno administrado con CPAP con una PEEP $4 \mathrm{cmH}_{2} \mathrm{O}$.

Durante los controles de constantes se observó unas lesiones en el brazo izquierdo (figura 1) delimitadas por el manguito de presión que nos alerta, por lo que decidimos realizar un seguimiento y estudio de la lesión. Una vez estabilizado el paciente se traslada al hospital de referencia para valoración nefrológica y posible diálisis debido a su Insuficiencia renal.

En el seguimiento al paciente en el hospital de referencia la lesión dérmica se extiende al resto de los miembros, tanto superiores como inferiores, lo que condujo a un estudio exhaustivo de la patología, Figura 1.- Imagen lesión brazo bajo la marca del manguito de presión.

la clínica, la presencia de eosinófilos y la realización de una biopsia, concluyendo con el diagnóstico de vasculitis Leucocitosclástica. 


\section{DISCUSIÓN}

Se plantean dos posibles hipótesis ante este fenómeno: La primera es la posibilidad de la realización de una mala praxis en la toma de la presión arterial, ya que el paciente había soportado la medida de la constante cada cinco minutos durante diez horas. No se ha encontrado bibliografía expresa que defina efectos secundarios a la técnica de la toma de esta constante, por lo que la hipótesis en la que la equimosis descrita es debida a la toma de presión arterial no ha podido evaluarse debido a la ausencia de estudios que la confirmen.

En cuanto a la segunda hipótesis, asumir la toma de la presión arterial seriada como responsable de una manifestación precoz de la equimosis como un síntoma de vasculitis Leucocitoclástica, requeriría de más estudios que lo confirmaran. No obstante nuestra experiencia nos orienta a que el éxtasis venoso podría haber facilitado y acelerado las reacciones mediadas por el sistema inmune que respondía al alérgeno, que en este caso era el antibiótico prescrito para su cistitis y disnea.

Para el diagnóstico de este tipo de vasculitis, además de la confirmación histopatológica, se requiere la realización de una analítica completa de sangre y orina para descartar afectación visceral. En éste caso, que es de origen farmacológico, tiene un excelente pronóstico. Sin embargo las vasculitis asociadas a enfermedades graves presentan una clínica más agresiva, con lesiones hemorrágicas, ampollas y úlceras ${ }^{9}$. El diagnóstico diferencial se debe hacer con la trombopenia, aunque en estos casos las lesiones purpúricas generalmente no son palpables, la coagulación intravascular diseminada (púrpura más o menos extensa, que puede ser o no palpable), el escorbuto (pápulas foliculares hemorrágicas en extremidades inferiores) y otras dermatosis purpúricas (macúlas purpúricas) similares.

Planteamos la necesidad de un estudio de posibles efectos secundarios a la toma de presión arterial; y de resultar realmente que no hay tales efectos, nos preguntamos si no podría ser este procedimiento útil para otros usos, como una detección precoz de 
problemas como la vasculitis y todos aquellos que presenten púrpuras. Si se hubieran contemplado estas lesiones como síntoma de vasculitis, hubiéramos podido dar un pronto tratamiento y resolución del problema, con ahorro del sufrimiento del paciente y del gasto sanitario. Además, en esta ocasión no era una vasculitis grave, pero de haberlo sido se habría podido tratar al paciente con prontitud para obtener un buen pronóstico. 


\section{BIBLIOGRAFÍA}

1. Instituto Social de la Marina, M. Guia sanitaria a bordo. (2007). at $<\mathrm{http}: / /$ www.segsocial.es/ism/gsanitaria_es/ilustr_capitulo7/cap7_2_hemorragias.htm>

2. Connor, C. W. in Clin. Anesth. (eds. Barash, P. G. et al.) (Lippincott Williams \& Wilkins, a Wolter Kluwer Business, 2013).

3. Love, B. R. T. The Tourniquet. ANZ J. Surg. 48, 66-70 (1978).

4. Swan, K. G., Wright, D. S., Barbagiovanni, S. S. \& Swan, B. C. Tourniquets revisited. J. Trauma 66, 672-5 (2009).

5. Miller, R. D., Fleisher, L. A., Savarese, J. J., Wiener-Kronish, J. \& Young, W. L. Miller's Anesthesia. (Elsevier Health Sciences, 2004).

6. Navarro, J. M. \& Perales, R. Guía práctica de enfermería en el paciente crítico (2 ${ }^{a}$ ed.). (Agencia Valenciana de Salud, Generalitat Valenciana, 2012).

7. Pérez C, L. et al. Edema hemorrágico agudo del lactante. Rev. Chil. pediatría 77, (2006).

8. Ardiles, L. G. \& Mezzano, S. A. Vasculitis Renales. Reumatologia 18, 4-9 (2002).

9. Rodríguez Peralto, J. L., Saiz, A. \& Segurado, A. in Dermatopatología: Correlación clínico-patológica (eds. Herrera, E., Moreno, A., Requena, L. \& Rodríguez, J. L.) 608 - 611 (Área Científica Menarini, 2007). 